\title{
Arbodiseases: a new and useful term for diseases related to arthropods
}

\author{
Carlos Brisola Marcondes ${ }^{[1]}$
}

[1]. Departamento de Microbiologia e Parasitologia, Universidade Federal de Santa Catarina, Florianópolis, SC, Brasil.

\section{Dear Editor:}

During the Cretaceous and Jurassic eras (145-65 MYA), blood feeding evolved at least six times in approximately 15,000 arthropod species in 400 genera. Four orders of insects include blood-feeders: Pthiraptera, Siphonaptera, Hemiptera, and Diptera. Some moths (Noctuidae) also feed on blood, and some chewing lice (Mallophaga) and flies with rasping structures in the tips of their probosces will possibly evolve to full blood feeding. All ticks (ca. 870 species in Acari: Ixodida) and several mites suck blood, and several ticks and mites may respectively cause paralysis and mange. Fly larvae can develop in or on the bodies of several animals. Several insects can transport microorganisms on their legs and in their intestinal tracts, which are then mechanically transmitted to other organisms. Some Arachnida (centipedes, spiders, scorpions, etc.) and hymenopteran (bees, wasps, and ants), heteropteran (true bugs) and other insects may cause envenomation.

By sucking blood, arthropods can cause direct damage, including allergy, and transmit many potentially pathogenic microorganisms, including more than 500 arboviruses ${ }^{1}$, bacteria, protozoa, and helminthes ${ }^{2}$. Although the modes of transmission are diverse, these potential pathogens have the common character of "hitchhiking" on/in arthropods, which protects them from environmental aggressions and allows them to be transported and distributed in efficient ways.

Diseases related to arthropods have been grouped as diseases transmitted and caused by arthropods, always needing the additional explanation that, in reality, arthropods transmit potential pathogens, and not diseases, which are a possible consequence of infection ${ }^{3}$. Other expressions are arthropod-borne diseases and arthropod borne diseases ${ }^{3}$, which are more generic and require no additional explanation.

Viruses transmitted by arthropods were initially called the mosquito-borne viral encephalitides, which was later changed to arthropod-borne virus encephalitides (when the involvement of ticks and Culicoides in several cycles became obvious) and arborviruses; finally being corrected to arboviruses, to prevent any association to trees (arbor in Latin) ${ }^{4}$. Similarly, the acronym arboterrorism was recently proposed to terrorism caused by arthropods ${ }^{5}$.

Because the utilization of arthropod borne diseases to represent all diseases in some way related to arthropods presents quite a mouthful and is somewhat cumbersome, I propose the adoption of arbodiseases, instead. The utilization of this word would facilitate communication and emphasize the relationship between these diseases and the need for preventive measures, besides stimulating comparative studies.

\section{REFERENCES}

1. International Committee on Taxonomy of Viruses (ICTV). Virus taxonomy: 2014 Release. http://www.ictvonline.org/virusTaxonomy. asp.

2. Lehane M. The Biology of Blood-Sucking in Insects. 2nd ed. Cambridge: Cambridge University Press; 2005. 321p.

3. Marcondes CB. Arthropod Borne Diseases. 1st ed. Cham: Springer International; 2017. 645p.

4. Reeves WC. Partners: serendipity in arbovirus research. J Vect Ecol. 2001;26(1):1-6.

5. Tabachnick WJ, Harvey WR, Becnel JJ, Clark GG, Connelly CR, Day JF, et al. Countering a bioterrorist introduction of pathogeninfected mosquitoes through mosquito control. J Am Mosq Control Assoc. 2011;27(2):165-7. 